\title{
Software defect detection based on selected complexity metrics using fuzzy association rule mining and defective module oversampling
}

\author{
Mohammad Farid Naufal \\ Department of Informatics \\ Universitas Surabaya \\ Surabaya, Indonesia \\ faridnaufal@staff.ubaya.ac.id
}

\author{
Selvia Ferdiana Kusuma \\ Department of Informatic \\ Institut Teknologi Sepuluh Nopember \\ Surabaya, Indonesia \\ selvia1805@mhs.its.ac.id
}

\begin{abstract}
Software defect is a major problem in software development. The cost of software development will be minimized when the software defects are detected earlier. Complexity metric is a mathematic calculation to calculate code complexity. It could be used to consider software defect detection. But, not all of complexity metrics influent on the occurrence of software defect, therefore it needs feature selection to select the most influent complexity metrics. Correlation-based Feature Selection (CFS) is used for selecting the most influent complexity metrics. This study conducted experiments on NASA Metric Data Program (MDP) datasets. NASA MDP contains software defect history logs based on several complexity metrics. But, there is an imbalanced distribution of defective and not defective modules in NASA MDP. The distribution of defective modules is less than not defective modules. It can reduce software defect detection performance. The distribution of defective module need to be reproduced. In this study, Synthetic Minority Oversampling Technique (SMOTE) is used to balance the distribution between defective and not defective modules. Software defect detection using Fuzzy Association Rule Mining (FARM) which is combined with the selection of complexity metrics using CFS and dataset balancing using SMOTE has sensitivity $85.51 \%$ and accuracy $91.63 \%$ in detecting software defective modules on NASA MDP dataset.
\end{abstract}

Index Terms - Software Defects, Fuzzy Association Rule Mining, CFS, SMOTE, Complexity Metric

\section{INTRODUCTION}

$\mathrm{D}$ eveloping good quality software is an expensive task. Minimizing the occurrence of software defect can also minimize the software development cost. Software defect detection is useful for software engineer to pay attention to defective modules (e.g. function, method, or classes) [1].

NASA MDP [2] are open source datasets which shows the occurrence of software defect module with its following software complexity metrics. NASA has publicly accessible datasets which is easy to compare this study with the previous researches which use the same dataset.

Complexity metric selection as attributes or features in software defect detection still be an issue today. Not all of complexity metric influent the occurrence of software defect [3]. It needs a method to select the most influential complexity metrics. CFS is used to analyze the attributes relation in classification [4]. It can be applied to select the most influential complexity metric in software defect detection.
The software defect distribution in training dataset is also important for detecting software defect. NASA MDP consist of two type distributions which are defective and not defective modules. If one of the distributions is more than the other, it causes the class imbalance. This situation can affect on software defect detection performance [5]. The majority class is the class which has higher distribution and the minority class is a class which has fewer distribution. Undersampling or oversampling technique can be used to solve this problem [6]. Undersampling is used for reducing the majority class and oversampling is used for increasing the minority class.

Defective modules distribution is less than not defective modules in NASA MDP dataset. It requires oversampling technique to increase defective modules distribution. SMOTE [7] is a technique to oversample the minority class. In this study SMOTE performs data oversampling on defective modules.

FARM is association rule mining extension with excess faster and more efficient in large quantitative dataset [8]. FARM generate the pattern of defective modules which is useful for detecting defective modules with complexity metric as attributes. FARM can be expected as a good approach to detect defective modules. This study proposes detection of software defect by applying FARM combined with attributes selection in complexity metric by applying CFS and defective modules oversampling using SMOTE.

\section{BACKGROUND}

\section{A. Related Work}

Burak [8] uses Branch Count, McCabe, and Halstead metric as features for software defect detection. CM1, JM1, $\mathrm{KC} 1, \mathrm{KC} 2$, and $\mathrm{PC} 1$ are NASA MDP dataset which are used in this study. This method is not considering the most influent complexity metrics to detect software defect but has fast computing time.

Ruchika [9] uses Support Vector Machine (SVM) to detect software defect on one CMI dataset in NASA MDP. This method uses Chidamber \& Kemerer (CK) Metrics [10] as features. But this study only tried on one dataset. Gabriela using relational association rule to detect software defect [11]. It uses CK metrics (Fan-in, Fan-Out, DIT, and NOC). 


\section{TABLE I. COMPLEXITY METRICS IN NASA MDP}

\begin{tabular}{ll}
\hline Symbol & Metric \\
\hline loc & line count of code \\
$\mathrm{v}(\mathrm{g})$ & cyclomatic complexity \\
$\mathrm{ev}(\mathrm{g})$ & essential complexity \\
$\mathrm{Iv}(\mathrm{g})$ & design complexity \\
$\mathrm{n}$ & total operators + operands \\
$\mathrm{v}(\mathrm{g})$ & Volume \\
$\mathrm{L}$ & program length \\
$\ldots$ & $\ldots$ \\
\hline
\end{tabular}

TABLE II. THE DESCRIPTION OF NASA MDP DATASET [2]

\begin{tabular}{ccl}
\hline Dataset & Language & \multicolumn{1}{c}{ Description } \\
\hline CM1 & $\mathrm{C}$ & $\begin{array}{l}\text { Instrument of } \\
\text { spacecraft }\end{array}$ \\
\hline JM1 & $\mathrm{C}$ & $\begin{array}{l}\text { Real-time predictor of ground } \\
\text { system: Generate predictions } \\
\text { using simulation. }\end{array}$ \\
\hline $\mathrm{KC1}$ & $\mathrm{C}++$ & $\begin{array}{l}\text { Management of storage for } \\
\text { processing and receiving } \\
\text { ground data }\end{array}$ \\
\hline $\mathrm{KC} 2$ & $\mathrm{C}++$ & $\begin{array}{l}\text { Another part of KC1 project. } \\
\text { Data processing of science. }\end{array}$ \\
\hline $\mathrm{PC} 1$ & $\mathrm{C}$ & $\begin{array}{l}\text { Flight application for earth } \\
\text { orbiting satellite }\end{array}$ \\
\hline
\end{tabular}

The weakness of this method is representing complexity metric. It converts complexity metric into Boolean relational association rule.

Omer Faruk [12] combine Artificial Bee Colony (ABC) and Artificial Neural Network (ANN) to detect software defect. Branch Count, McCabe, and Halstead metric are used as features. The NASA MDP datasets which are used in this study are CM1, JM1, KC1, KC2, and PC1. This method is easy to implement in quantitative complexity metrics values. But it's performance is not significant while compared with other software defect detection method.

\section{B. Complexity Metrics}

NASA MDP provides the occurrence of software defect with related complexity metrics. These complexity metrics can be used as features for software defect detection. Branch Count, Halstead metric [13], and McCabe metrics [14] are widely used software metric which represent the code complexity. Table 1 shows list of some complexity metrics.

\section{Correlation-based Features Selection}

A useful feature is a feature which is correlated with predicted class, otherwise it is irrelevant feature [4]. Correlation-based feature selection select the most influent features by considering the correlation between featuresfeatures and features-predicted class. The calculation of CFS evaluation is shown in Eq. (1).
$M_{S}=\frac{k \overline{r_{c f}}}{\sqrt{k+k(k-1) \overline{r_{f f}}}}$

$M_{S}$ is a Merit or heuristic value of $\mathrm{S}$ which containing $\mathrm{k}$ features. $\overline{r_{c f}}$ is value of average correlation between predicted classes and features $(\mathrm{f} \in \mathrm{S}), \overline{r_{f f}}$ is value of average correlation between features and other features. Rank of the most influent metric to predict class is shown in Eq. (1). Value of $\overline{r_{c f}}$ and $\overline{r_{f f}}$ are calculated by using the Symmetrical Uncertainty $(S U)$. The equation of $S U$ is shown in Eq. (2).

$S U=2.0 \times\left[\frac{H(X)-H(Y \mid X)}{H(Y)+H(X)}\right]$

The entropy $\mathrm{H}(\mathrm{X})$ and $\mathrm{H}(\mathrm{Y})$ are the possible emergence of an attribute $(x \in X)$ or $(y \in Y)$ in a transaction. The calculation of $\mathrm{H}(\mathrm{X}), \mathrm{H}(\mathrm{Y})$, and $\mathrm{H}(\mathrm{X} \mid \mathrm{Y})$ are shown in Eq. (3), (4), and (5).

$$
\begin{aligned}
& H(Y)=-\sum_{(y \in Y)} p(y) \log _{2}(p(y)) \\
& H(X)=-\sum_{(x \in X)} p(x) \log _{2}(p(x)) \\
& H(X \mid Y)=-\sum_{(x \in X)} p(x) \sum_{(y \in Y)} p(y \mid x) \log _{2}(p(y \mid x))
\end{aligned}
$$

\section{Synthetic Minority Oversampling Technique}

SMOTE solve the class imbalanced problem by reproducing or oversampling of the minority class distribution [7]. Oversampling is the way to create synthetic data by merging $\mathrm{k}$ nearest neighbors of minority class samples. K-nearest neighbors is randomly selected depend on the amount of desired synthetic data. Synthetic data in software defective modules is formed in the following way:

a. Select one of defective module randomly.

b. Take the k-nearest neighbors of that defective module based on its complexity metric value.

c. Randomly select $\mathrm{n}$ of selected defective modules.

d. Calculate the differences value between selected defective module's complexity metric with its $\mathrm{N}$ nearest neighbors.

e. Multiply a random value from $[0,1]$ with previous differences value.

f. Add the selected defective module's complexity metric with the multiplied differences from step e. Eq. (6) is the calculation to form oversampled defective module. $N, a$, and $b$ are vector of defective module which consist of complexity metrics m. $N$ is oversampled dataset vector $N\left(\mathrm{~m}_{\mathrm{n} 1}, \mathrm{~m}_{\mathrm{n} 2}, \mathrm{~m}_{\mathrm{n} 3}, \ldots\right), a$ is selected defective module vector a $\left(\mathrm{m}_{\mathrm{a} 1}, \mathrm{~m}_{\mathrm{a} 2}, \mathrm{~m}_{\mathrm{a}}\right.$, $\ldots), r$ is random value $[0,1]$, and $b$ is one of nearest neighbor vector of $a, b\left(\mathrm{~m}_{\mathrm{b} 1}, \mathrm{~m}_{\mathrm{b} 2}, \mathrm{~m}_{\mathrm{b} 3}, \ldots\right)$.

$N=a+r(a-b)$

\section{FUZZY ASSOCIATION RULE MINING}

FARM [15] is Association Rule Mining (ARM) expansion. ARM represent the appearance of item in transaction as a Boolean value. In FARM represent the data as a membership function between 0 and 1. FARM is a good method for detecting software defect which has quantitative complexity metric attributes. 
TABLE III. SOFTWARE DEFECT HISTORY LOGS IN NASA MDP DATASET

\begin{tabular}{cccccccc}
\hline ID & loc & $\mathbf{v}(\mathbf{g})$ & ev(g) & iv(g) & n & $\begin{array}{c}\text { other } \\
\text { metrics }\end{array}$ & $\begin{array}{c}\text { defect } \\
\text { status }\end{array}$ \\
\hline $\mathbf{1}$ & 24 & 5 & 1 & 3 & 63 & $\ldots$ & FALSE \\
$\mathbf{2}$ & 20 & 4 & 4 & 2 & 47 & $\ldots$ & FALSE \\
$\mathbf{3}$ & 31 & 4 & 1 & 2 & 141 & $\ldots$ & TRUE \\
$\mathbf{4}$ & 29 & 5 & 1 & 3 & 111 & $\ldots$ & TRUE \\
\hline
\end{tabular}

$$
\begin{aligned}
& \operatorname{Supp}(A, B)=\frac{\text { All transactions contain } A \text { and } B}{\text { All transactions }} \\
& \operatorname{Conf}(A, B)=\frac{\text { All transactions contain } A \text { dan } B}{\text { All transactions contains } A}
\end{aligned}
$$

There are several methods for finding association rules in fuzzy sets. F-APACS [16] describes quantitative attributes into fuzzy sets by using a linguistic term. Apriori [17] performs fuzzification of quantitative attributes then categorize them into membership functions. Finally, association rules are generated using fuzzy sets. But, Apriori efficiency is not good enough. Han [18] propose FrequentPattern growth (FP-Growth) as a new mining method to improve Apriori efficiency. This method more efficient because it does not generate all item set candidates. FPGrowth categorize every transaction into Frequent Pattern tree (FP-Tree). Then it calculates the support and confidence for every pattern. Eq. (7) and Eq. (8) show support and confidence calculation.

\section{Methodology}

The detail of this study approaches will be described in this section.

\section{A. Collecting Dataset}

NASA MDP dataset are used for evaluating our method. NASA MDP datasets contain several software defect history logs based on Branch Count, McCabe, and Halstead metric. This dataset publicly accessible and used in many previous researches.

This study uses 5 NASA MDP datasets which are CM1, $\mathrm{JM} 1, \mathrm{KC} 1, \mathrm{KC} 2$, and PC1. Table 2 shows the description of each dataset and Table 3 shows the software defect history logs in NASA MDP dataset. Every row is a module, it could be a method or a function and every column is a complexity metric calculation for each module. The rightmost column is a defective status for each module. For example, at the first row is a method with ID 1, it has 24 line of codes (loc), 5 cyclomatic complexity $(\mathrm{v}(\mathrm{g})), 1$ essential complexity $(\mathrm{ev}(\mathrm{g}))$, 3 design complexity (iv(g)), 63 total operators and operands (n), and defective status of that module is false. Another example at the third row is a method with id 3, it has 31 line of codes (loc), 4 cyclomatic complexity $(\mathrm{v}(\mathrm{g}))$, 1 essential complexity (ev(g)), 2 design complexity (iv(g)), 141 total operators and operands (n), and defective status of that module is true.

\section{B. Preprocessing}

In the preprocessing step, CFS and SMOTE are used to select the most influent complexity metric and oversample the training dataset. From 21 complexity metrics on NASA MDP

\section{TABLE IV RANDOMLY SELECTED DEFECTIVE MODULE}

\begin{tabular}{cccc}
\hline ID & ev(g) & b & IOComment \\
\hline 1 & 32 & 2 & 0.43 \\
\hline
\end{tabular}

TABLE V RANDOMLY 5 NEAREST NEIGHBORS

\begin{tabular}{cccc}
\hline ID & ev(g) & b & IOComment \\
\hline 1 & 41 & 6 & 0.33 \\
$\mathbf{2}$ & $\mathbf{2 3}$ & $\mathbf{5}$ & $\mathbf{0 . 0 8}$ \\
$\mathbf{3}$ & $\mathbf{3 8}$ & $\mathbf{5}$ & $\mathbf{0 . 3 5}$ \\
$\boldsymbol{\cdots}$ & $\boldsymbol{\cdots}$ & $\boldsymbol{\cdots}$ & $\boldsymbol{\cdots}$ \\
\hline \multicolumn{4}{c}{ TABLE VI. NEW DEFECTIVE MODULE FROM } \\
\multicolumn{5}{c}{ OVERSAMPLING PROCESS } \\
\hline ID & ev(g) & b & IOComment \\
\hline N2 & 33.8 & 1.4 & 0.5 \\
N5 & 30.2 & 1.1 & 0.454 \\
\hline
\end{tabular}

dataset, CFS will select the most influent metric for detecting defective modules on each dataset. Good complexity metric combinations selection is based on merit value on Eq. 1. The best complexity metric combination has the highest merit value. CFS and SMOTE are executed using WEKA tools [19]. SMOTE will reproduce the defective modules on training dataset. This phase has been completed after selection of complexity metric using CFS. The example of oversampling process are as follows:

a. Table 4 shows one of randomly selected defective module which will be oversampled.

b. Select the k nearest neighbors of Table 4 randomly. Table 5 shows the 5 randomly nearest neighbors of Table 4 and ID 2 and 5 are selected nearest neighbors.

c. Calculate oversampled defective module using Eq (6) as follows:

$N 2=(32,2,0.43)+0.2 x[(32,2,0.43)-(23,5,0.08)]$

$N 5=(32,2,0.43)+0.3 x[(32,2,0.43)-(38,5,0.35)]$

$N 2$ and $N 5$ are new defective modules from oversampling process of selected defective module on Table 4. Table 6 shows the new defective module of N1 and $\mathrm{N} 2$.

\section{Training}

In this step, software defect pattern will be obtained. FP Growth is used for conducting training step. Then FP-Tree is obtained based on complexity metrics. The detail of training steps are as follows:

\section{1) Determining Fuzzy Membership Function}

Each complexity metric of defective modules will be categorized its fuzzy membership function value. The kind of fuzzy membership functions are trapezoidal, triangular, gaussian function. This study uses triangular fuzzy membership function because it can more precisely write the more and less value [20] in this case complexity metric. The membership functions are "low", "medium", and "high". The $\mathrm{x}$-axis is complexity metric value and $\mathrm{y}$-axis is membership value of complexity metric to each category.

The limit value of $a, b$, and $c$ for every complexity metric in training dataset defines as:

$\mathrm{y}=$ complexity metric value 
$\mathrm{a}=$ the minimum value of $\mathrm{y}$

$\mathrm{b}=\mathrm{y}$ mean value

$\mathrm{c}=2 \mathrm{x}$ y mean value

2) Mapping Complexity Metric to Fuzzy Membership

In this step the membership function of complexity metric $\mu(\mathrm{x})$ will be calculated in each category (low, medium, and high). In Table 7 , the complexity metric has been converted into three categories (low, medium, and high) fuzzy membership. For example, at the first row, a module with ID 1 has low of ev(g) and low medium of $b$ and low medium of IOComment. $b$ and IOComment are metric which have two fuzzy memberships. Low medium means that complexity metric has two fuzzy memberships which are low and medium.

\section{3) Identify Defective Patterns}

The defective pattern will be identified by using FP Growth. The next step is calculating each defective pattern confidence. Then this pattern will be used for detecting defective modules. Table 8 shows the defective pattern based on complexity metric which are generated from training step. For example at the first row the defective pattern with ID 1 is ev(g).Low, ev(g).Med, b.High, 1OComment.High and has confidence value 1 . It means the module which has low medium of ev(g) and high of $\mathrm{b}$ and high of IOComment has $100 \%$ probability to be a defective module. Another example at the third row the defective pattern with ID 3 is ev(g).Low, ev(g).Med, b.Med, b.High, lOComment.Med, 1OComment.High and has confidence value 0.875 . It means the module which has low medium of ev(g) and medium high of $b$, and medium high of IOComment has $87.5 \%$ probability to be a defective module.

\section{Defective Modules Detection}

The generated defective modules patterns from training step will be used to detect defect on testing dataset. A module is detected as defective module if it is matched with one of defective modules pattern and vice versa.

\section{E. Performance Evaluation}

True Positive (TP) is the defective module number which successfully detected as defect, True Negative (TN) is the not defective module number which successfully detected as not defect, False Positive (FP) is the not defective module number which classified as defect, False Negative (FN) is the defective modules number which classified as defect. Accuracy, sensitivity, specificity, precision, nad probability of false alarm are used as performance evaluation.

\section{F. Results}

This study has been done on five NASA MDP datasets. Testing scenario has been done without oversampling (FARM-CFS) and with oversampling process (FARM-CFSSMOTE). This scenario is done to compare the effect of oversampling on defect detection performance. This proposed approach use weka to run CFS and SMOTE.

We are dividing the dataset randomly into two parts, $50 \%$ for training and $50 \%$ for testing. The reason is we assume in real software project, the model of defect detection is formed in the middle of software construction. We also compare the accuracy, sensitivity, precision, specificity and probability of false alarm (pof) of this detection approach with another detection method using Naïve Bayes (NB), Support Vector Machine (SVM), Relational Association Rule
(RAR), Artificial Neural Network + Artificial Bee Colony $(\mathrm{ANN}+\mathrm{ABC})$.

Table 9 shows the selected metric of each dataset. From 21 complexity metrics in Table 1, CFS select only 3 complexity metrics on $\mathrm{CM} 1,7$ on $\mathrm{JM} 1,8$ on $\mathrm{KC} 1,8$ on $\mathrm{KC} 2$, and 3 on PC1. It can be seen that IOComment is the most influent metric in all dataset, followed by locCodeAndComment and $\mathrm{ev}(\mathrm{g})$ which are influent metric in 3 datasets, IOBlank, uniqOp, iv(g), loc, b, and I are influent metric at least in 2 datasets.

The result of this approach can be clearly seen on Table 10. Table 10 shows the performance average of FARM-CFS and FARM-CFS-SMOTE at every confidence threshold value.

Accuracy comparison between FARM-CFS and FARMCFS-SMOTE is not significant but when the confidence threshold above 0.3 FARM-CFS-SMOTE is better than FARM-CFS. This indicate that when confidence threshold is above 0.3 , the defective pattern of FARM-CFS-SMOTE can detect more defective pattern as a defect than detecting not defective pattern as defect. In other words FARM-CFSSMOTE has more true positive and true negative value than FARM-CFS.

Specificity comparison between FARM-CFS and FARM-CFS-SMOTE are significant when the confidence value under 0.3 , it is caused by true negative value of FARMCFS is always better than FARM-CFS-SMOTE. Moreover defective pattern which formed by oversampling process is not always accurate to detect defective modules. In another word FARM-CFS-SMOTE has more false positive value than FARM-CFS.

Sensitivity comparison between FARM-CFS and FARM-CFS-SMOTE are significant. FARM-CFS-SMOTE sensitivity is better than FARM-CFS at every confidence threshold. It caused by oversampling process which reproduce more defective pattern can detect more defective module. In other word, FARM-CFS-SMOTE has more true positive value.

Precision comparison between FARM-CFS and FARMCFS-SMOTE are significant when confidence threshold above 0.6 . It is caused by true positive value of FARM-CFSSMOTE more than FARM-CFS compared by the false positive value.

Probability of false alarm comparison between FARMCFS and FARM-CFS-SMOTE are not significant but FARM-CFS is better at every confidence threshold. It is caused by defective pattern which is formed by oversampling process sometimes detect not defective modules as defect.

The comparison of these two scenarios can be clearly seen on Table 10. It shows the comparison of the best value of each performance evaluation at each confidence threshold. FARM-CFS has better specificity, precision, and probability of false alarm than FARM-CFS-SMOTE by a margin of each are $9.35 \%, 1.14 \%$, and $1.32 \%$. However, the imbalance of defective and not defective modules in the dataset make detecting the defective modules is more important. FARMCFS-SMOTE which has a higher sensitivity value still better assessed because it more able to detect more defective modules. In other words FARM-CFS-SMOTE has more true positive value than FARM-CFS.

Table 11 shows the performance comparison between this approach with NB, SVM, RAR, and ANN+ABC. FARM-CFS-SMOTE has the highest sensitivity than the NB, SVM, RAR, ANN + ABC, and FARM-CFS. Although the 


\begin{tabular}{|c|c|c|c|c|c|c|c|c|c|c|}
\hline \multirow{2}{*}{ ID } & \multicolumn{3}{|c|}{ ev(g) } & \multicolumn{3}{|c|}{ b } & \multicolumn{3}{|c|}{ IOComment } & \multirow{2}{*}{$\begin{array}{l}\text { Defect } \\
\text { Status }\end{array}$} \\
\hline & Low & Medium & High & Low & Medium & High & Low & Medium & High & \\
\hline 1 & 1 & 0 & 0 & 1 & 1 & 0 & 1 & 1 & 0 & FALSE \\
\hline 2 & 1 & 0 & 0 & 1 & 1 & 0 & 1 & 0 & 0 & FALSE \\
\hline 3 & 1 & 0 & 0 & 1 & 1 & 0 & 1 & 0 & 0 & FALSE \\
\hline
\end{tabular}

TABLE VIII. THE DEFECTIVE PATTERN BASED ON COMPLEXITY METRIC

\begin{tabular}{llc}
\hline ID & Defective Pattern & Confidence \\
\hline 1 & ev(g).Low, ev(g).Med, b.High, lOComment.High & 1 \\
2 & ev(g).Low, ev(g).Med, b.High, lOComment.Med, 1OComment.High & 1 \\
3 & ev(g).Low, ev(g).Med, b.Low, b.Med, 1OComment.Low, 1OComment.Med & 0.844 \\
\hline
\end{tabular}

TABLE IX. SELECTED COMPLEXITY METRIC OF NASA MDP DATASET

\begin{tabular}{lllll}
\hline CM1 & JM1 & KC1 & KC2 & PC1 \\
\hline ev(g) & loc & ev(g) & IOComment & i \\
$\mathrm{b}$ & $\mathrm{v}(\mathrm{g})$ & $\mathrm{iv}(\mathrm{g})$ & IOCodeAndComment & IOComment \\
IOComment & $\mathrm{ev}(\mathrm{g})$ & $\mathrm{v}(\mathrm{g})$ & IOBlank & IocCodeAndComment \\
& $\mathrm{iv}(\mathrm{g})$ & IOCode & uniq_Op & loc \\
& $\mathrm{e}$ & IOComment & loc & $\mathrm{ev}(\mathrm{g})$ \\
& IOComment & IOBlank & ev(g) & $\mathrm{i}$ \\
& locCodeAndComment & uniq_Op & $\mathrm{i}$ & $\mathrm{b}$ \\
& & total_Opnd & $\mathrm{b}$ & \\
\hline
\end{tabular}

TABLE X. PERFORMANCE COMPARISON BETWEEN FARM-CFS AND FARM-CFS-SMOTE

\begin{tabular}{cccccc|ccccc}
\hline \multirow{2}{*}{ Confidence } & \multicolumn{5}{c}{ FARM-CFS } & \multicolumn{5}{c}{ FARM-CFS-SMOTE } \\
\cline { 2 - 11 } & Accuracy & Specificity & Sensitivity & Precision & Pof & Accuracy & Specificity & Sensitivity & Precision & Pof \\
\hline 0.1 & $87.96 \%$ & $90.15 \%$ & $\mathbf{6 9 . 5 6 \%}$ & $55.61 \%$ & $9.82 \%$ & $84.08 \%$ & $83.61 \%$ & $\mathbf{8 5 . 5 1 \%}$ & $48.57 \%$ & $16.36 \%$ \\
0.2 & $88.15 \%$ & $95.31 \%$ & $54.18 \%$ & $71.83 \%$ & $4.66 \%$ & $85.74 \%$ & $86.27 \%$ & $82.31 \%$ & $51.91 \%$ & $13.70 \%$ \\
0.3 & $87.57 \%$ & $95.64 \%$ & $47.29 \%$ & $72.65 \%$ & $4.34 \%$ & $90.18 \%$ & $92.98 \%$ & $70.74 \%$ & $63.36 \%$ & $6.99 \%$ \\
0.4 & $89.00 \%$ & $96.17 \%$ & $38.20 \%$ & $\mathbf{7 5 . 8 6 \%}$ & $3.81 \%$ & $89.94 \%$ & $94.07 \%$ & $62.87 \%$ & $65.56 \%$ & $5.90 \%$ \\
0.5 & $88.24 \%$ & $96.55 \%$ & $30.96 \%$ & $73.03 \%$ & $3.42 \%$ & $\mathbf{9 0 . 4 1 \%}$ & $96.03 \%$ & $54.62 \%$ & $72.54 \%$ & $3.95 \%$ \\
0.6 & $89.11 \%$ & $99.09 \%$ & $21.77 \%$ & $46.66 \%$ & $0.90 \%$ & $89.95 \%$ & $\mathbf{9 6 . 5 7 \%}$ & $45.54 \%$ & $74.06 \%$ & $3.41 \%$ \\
0.7 & $\mathbf{8 9 . 1 3 \%}$ & $99.73 \%$ & $19.08 \%$ & $54.94 \%$ & $0.26 \%$ & $89.73 \%$ & $96.57 \%$ & $42.40 \%$ & $\mathbf{7 4 . 7 2 \%}$ & $3.21 \%$ \\
0.8 & $88.41 \%$ & $99.76 \%$ & $15.46 \%$ & $55.25 \%$ & $0.24 \%$ & $89.78 \%$ & $97.37 \%$ & $39.47 \%$ & $75.93 \%$ & $2.60 \%$ \\
0.9 & $88.40 \%$ & $\mathbf{9 9 . 7 6 \%}$ & $15.39 \%$ & $55.23 \%$ & $\mathbf{0 . 2 4 \%}$ & $88.90 \%$ & $98.41 \%$ & $26.24 \%$ & $72.35 \%$ & $\mathbf{1 . 5 6 \%}$ \\
\hline
\end{tabular}

accuracy, specificity, precision, and probability of false alarms FARM-CFS-SMOTE is not the best, but sensitivity is the priority in defect detection. The higher value of sensitivity, it is better in detecting defective modules that have lower distribution in the dataset. The imbalance distribution of defective and not defective modules makes detection of defective modules which have lower distribution becomes more essential.

\section{G. Conclusion}

From the results, it can be concluded that the addition of oversampling process on defective modules can significantly increase the sensitivity up to $15.96 \%$ compared with no oversampling process. Although the oversampling process does not improve accuracy significantly, it is only $1.18 \%$ compared without oversampling process.
FARM-CFS-SMOTE has the best sensitivity compared with SVM, NB, RAR, ANN+ABC, and FARM-CFS. It means that FARM-CFS-SMOTE can detect more defective modules than the other methods. The lower distribution of defective modules compared with not defective modules make detection of defective module is more essential. The defective pattern which generated from oversampling process can detect the defective modules correctly. Although several not defective modules are classified as defect (false positive) but the value is not significant if compared with defective modules which are classified as defect (true positive). Software defect detection performed in this study is based on complexity metrics McCabe, Halstead, and Branch Count at NASA MDP dataset. 
TABLE XI. PERFORMANCE COMPARISON WITH ANOTHER CLASSIFICATION METHOD

\begin{tabular}{llllll}
\hline $\begin{array}{c}\text { Defect } \\
\text { Detection } \\
\text { Method }\end{array}$ & Accuracy & Specificity & Sensitivity & Precision & Pof \\
\hline NB & $87.95 \%$ & $95.17 \%$ & $50.10 \%$ & $71.86 \%$ & $4.84 \%$ \\
\hline SVM & $\mathbf{9 2 . 4 8 \%}$ & $\mathbf{9 9 . 9 5 \%}$ & $54.36 \%$ & $\mathbf{9 9 . 2 4 \%}$ & $\mathbf{0 . 0 4 \%}$ \\
\hline RAR & $87.69 \%$ & $89.33 \%$ & $82.80 \%$ & $69.83 \%$ & $10.70 \%$ \\
\hline ANN+ABC & $69.60 \%$ & $56.41 \%$ & $76.60 \%$ & $22.55 \%$ & $29.80 \%$ \\
\hline $\begin{array}{l}\text { FARM- } \\
\text { CFS }\end{array}$ & $91.09 \%$ & $99.76 \%$ & $69.56 \%$ & $93.54 \%$ & $0.24 \%$ \\
\hline $\begin{array}{l}\text { FARM- } \\
\text { CFS- } \\
\text { SMOTE }\end{array}$ & $91.63 \%$ & $98.41 \%$ & $\mathbf{8 5 . 5 1 \%}$ & $80.35 \%$ & $1.56 \%$ \\
\hline
\end{tabular}

\section{H. Future Work}

In the future software defect detection can be developed and applied to another software development project. Furthermore, determining the confidence threshold value can be detailed in smaller units to get the better performance.

\section{REFERENCES}

[1] P. He and B. Li, "An empirical study on software defect prediction with a simplified metric set," Information and Software Technology, vol. 59, pp. 170-190, 2015.

[2] NASA, "PROMISE," University of Ottawa, 2006. [Online]. Available: http://promise.site.uottawa.ca/SERepository/datasetspage.html. [Accessed 3 May 2015].

[3] V. T. A. S. Ishani Arora, "Open Issues in Software Defect Prediction," Procedia Computer Science, pp. 906-912, 2015.

[4] M. A. Hall, "Correlation-based feature selection for machine learning," 1998.

[5] M. Galar, A. Fernández, E. Barrenechea, H. Bustince and F. Herrera, "A Review on Ensembles for the Class Imbalance Problem: Bagging, Boosting-, and Hybrid-Based Approaches," Systems, Man, and Cybernetics, Part C: Applications and Reviews, IEEE Transactions, pp. 463-484, 2012.

[6] R. Barandela, R. Valdovinos, J. Sánchez and F. Ferri, "The imbalanced training sample problem: Under or over sampling?," Structural, Syntactic, and Statistical Pattern Recognition, pp. 806814, 2004.

[7] Chawla, N. V. a. Bowyer, K. W. a. Hall, L. O. a. Kegelmeyer and W. Philip, "SMOTE: Synthetic Minority Over-sampling Technique," CoRR, pp. 321--357, 2002.

[8] B. Turhan and A. Bener, "Analysis of Naive Bayes assumptions on software fault data: An empirical study," Data \& Knowledge Engineering, pp. 278-290, 2009.

[9] R. Malhotra, "A systematic review of machine learning techniques for software fault prediction," Applied Soft Computing, vol. 27, p. 504-518, 2015.

[10] M. Hitz and B. Montazeri, "Chidamber and Kemerer's metrics suite: a measurement theory perspective," in IEEE Transactions on Software Engineering, 1996.

[11] G. Czibula, "Software defect prediction using relational association rule," Information Sciences, pp. 260-278, 2014.

[12] N. Omar, S. S. Haris, R. Hassan, H. Arshad, M. Rahmat, N. F. A Zainal and R. Zulkifli, "Automated Analysis of Exam Questions According to Bloom's Taxonomy," Science Direct, pp. 297-303, 2012.

[13] M. H. Halstead, "Elements of Software Science (Operating and Programming Systems Series," 1977.

[14] T. McCabe, "A Complexity Measure," Software Engineering, IEEE Transaction, Vols. SE-2, pp. 308-320, 1976.

[15] C.-H. Wang and W.-H. Lee, "Applying Fuzzy FP-Growth to Mine Fuzzy Association Rules," 2010
[16] W. Au and K. Chan, "An effective algorithm for discovering fuzzy rules in relational databases," in IEEE World Congress on Computational Intelligence, 1998.

[17] A. Inokuchi, T. Washio and H. Motoda, "An Apriori-Based Algorithm for Mining Frequent Substructures from Graph Data," in Principles of Data Mining and Knowledge Discovery, 2002.

[18] H. Jiawei, P. Jian and Y. Yiwe, "Mining Frequent Patterns Without Candidate Generation," SIGMOD Rec., vol. 29, no. 2, pp. 1-12, 2000.

[19] Hall, M. a. Frank, E. a. Holmes, G. a. Pfahringer, B. a. Reutemann, P. a. Witten and I. H, "The WEKA Data Mining Software: An Update," SIGKDD Explor. Newsl., vol. 11, pp. 10-18, 2009.

[20] A. K. Shyamal and M. Pal, "Triangular Fuzzy Matrices," Iranian Journal of Fuzzy Systems, vol. 4, no. 1, pp. 75-87, 2007. 
2019 16th International Joint Conference on Computer Science and Software Engineering (JCSSE 2019)

Chonburi, Thailand 10 - 12 July 2019 
Copyright (C) 2019 by the Institute of Electrical and Electronics Engineers, Inc. All Rights Reserved

Copyright and Reprint Permissions: Abstracting is permitted with credit to the source. Libraries are permitted to photocopy beyond the limit of U.S. copyright law for private use of patrons those articles in this volume that carry a code at the bottom of the first page, provided the per-copy fee indicated in the code is paid through Copyright Clearance Center, 222 Rosewood Drive, Danvers, MA 01923.

For other copying, reprint or republication permission, write to IEEE Copyrights Manager, IEEE Service Center, 445 Hoes Lane, Piscataway, NJ 08854. All rights reserved.

*** This is a print representation of what appears in the IEEE Digital Library. Some format issues inherent in the e-media version may also appear in this print version.

$\begin{array}{ll}\text { IEEE Catalog Number: } & \text { CFP1932P-POD } \\ \text { ISBN (Print-On-Demand): } & 978-1-7281-0720-2 \\ \text { ISBN (Online): } & 978-1-7281-0719-6 \\ \text { ISSN: } & 2372-1642\end{array}$

\section{Additional Copies of This Publication Are Available From:}

Curran Associates, Inc

57 Morehouse Lane

Red Hook, NY 12571 USA

Phone: (845) 758-0400

Fax: $\quad$ (845) 758-2633

E-mail:_curran@proceedings.com

Web: $\quad$ www.proceedings.com 


$\begin{array}{lll}\text { Keynote } & \text { Title } & \text { Page }\end{array}$

$\begin{array}{lll}\text { Keynote-I Business Transformation with Blockchain } & \text { XXIV }\end{array}$ by Professor Dr.Dusit Niyato

Keynote-II Matching Next-Gen HPC with Target Applications $\quad$ XXV by Professor Dr. Pascal Bouvry

$\begin{array}{lll}\text { Paper ID } & \text { Title Page }\end{array}$

1570538175 Hybrid EEG-fEMG based Human-Machine Interface for 1 Communication and Control Applications

by Kessarabhorn Chuysud and Yunyong Punsawad

1570547231 Circular Vector Field Analysis for the Adaptive Diffusion Flow Snakes Applied to Ultrasound Images of Breast Cancer

by Annupan Rodtook and Khwunta Kirimasthong

1570547324 A Linear-time Algorithm for Optimal Tree Completion

by Chawin Aiemvaravutigul and Nonthaphat Wongwattanakij

1570537927 Thai Handwriting Beautification

by Supawan Tasanaprasert and Karun Tonmaithong

1570528296 Discovering Factors Associated with Online Gaming Behaviors

by Bernardinus Harnadi

1570529455 Enhancing a Keyword Search Using Segmentation and Similarity Measure Algorithms : A Case Study of Phuket Attractions

by Kitsiri Chochiang and Witaya Khuanwilai

1570537320 ARCode: Augmented Reality Application for Learning Elementary Computer Programming

by Sirawit Sittiyuno and Kornchawal Chaipah 


\section{$\begin{array}{lll}\text { Paper ID } & \text { Title } & \text { Page }\end{array}$}

1570542545 Web-based Elderly Monitoring System with GIS 38

by Anirut Sriwichian, Veera Boonjing, Jirapond Muangprathub and Pichetwut Nillaor

1570542664 A Result Verification of Decision Tree Model for Industrial

Wireless Sensors Selection using Analytic Hierarchy Process

by Saksiri Meesawad, Bundit Thanasopon

and Olarn Wongwirat

1570542733 An Ontology for SNORT Rule

by Assadarat Khurat and Wudhichart Sawangphol

1570542789 An Information Integration System to Continuing of Care

Case study Nongsung Hospital, Mukdahan THAILAND

by Pranithan Klangprapunt and Pusadee Seresangtakul

1570542908 WhatTheHealth: An Android Application for Consumers of Healthy Food

by Songsri Tangsripairoj, Nonthpat Wongkham,

Bongkotmanee Leelalerkiat and Sarun Chuenpukdi

1570543015 Game Elements to Promote Walking in Thais Working Adults

by Sakchai Muangsrinoon and Poonpong Boonbrahm

1570543074 Condition Based Maintenance for Data Center Operations Management

by Montri Wiboonrat

1570547326 Practical Differential Privacy for Location Data Aggregation using a Hadamard Matrix

by Patinya Sangiamchit and Jittat Fakcharoenphol

1570542774 IVAA: Intelligent Vehicle Accident Analysis System

by Kundjanasith Thonglek, Norawit Urailertprasert,

Patchara Pattiyathanee and Chantana Chantrapornchai 


\section{$\begin{array}{lll}\text { Paper ID } & \text { Title } & \text { Page }\end{array}$}

1570542836 DATA++: An Automated Tool for Intelligent Data Augmenta- 91 tion Using Wikidata

by Waran Taveekarn, Chatchanin Yimudom, Supisara Sukkanta, Steven Lynden, Wudhichart Sawangphol and Suppawong Tuarob

1570542940 Multi-Paths Generation for Structural Rule Quests

by Thongtham Chongmesuk and Vishnu Kotrajaras

1570542954 Kiddy Manner: A Game-Based Mobile Application for

Children Learning Thai Social Etiquette

by Songsri Tangsripairoj, Mathawee Sukkhet, Jidapa Sumanotham and Benya Yusuk

1570543067 Speech-to-Thai Sign Language Conversion for Thai Deaf: A

by Nattapol Namyang, Jarukit Lumpaolertwilai and

Suphakant Phimoltares

1570543079 Semi-Automatic Word-Aligned Tool for Thai-Vietnamese Parallel Corpus Construction

by Dang Ngoc Chuong and Pusadee Seresangtakul

1570527283 Development of Reliable Wireless Communication System for Secure Blockchain-based Energy Trading

by Zhuoxian Huang, Kongrath Suankaewmanee, Jiawen Kang, Dusit Niyato and Pei Sin Ng

1570542701 An In-Memory Checkpoint-Restart Mechanism for a Cluster of Virtual Machines

by Jumpol Yaothanee and Kasidit Chanchio

1570542745 Moving Object Detection using Integrated Spatial and Motion-Based Method

by Manit Chansuparp and Kulsawasd Jitkajornwanich

1570543979 Estimating the new Initial Value of Trial Division Algorithm for Balanced Modulus to Decrease Computation Loops

by Kritsanapong Somsuk, Thanapat Chiawchanwattana and Chalida Sanemueang 


\section{$\begin{array}{lll}\text { Paper ID } & \text { Title } & \text { Page }\end{array}$}

1570546542 A DIFF-Based Indoor Positioning System Using Fingerprinting 142

Technique and K-Means Clustering Algorithm by Apichon Anuwatkun, Jirapat Sangthong and Sommart Sang-Ngern

1570547233 Enhanced DDoS Detection using Hybrid Genetic Algorithm 146 and Decision Tree for SDN

by Parinya Preamthaisong, Anucha Auyporntrakool,

Phet Aimtongkham, Titaya Sriwuttisap and Chakchai So-In

1570542673 The Control Model for Environmental Factor Effecting on by Narongsak Lekbangpong, Theera Srisawa, Apirat Wanichsombat and Jirapond Muangprathu

1570543106 A Low-Cost RTK GNSS Receiver with Cloud-Based Control Center Application by Duangduen Asavasuthirakul, Sittha Saisawan, Antony Harfield and Prasert Wiangsukphaiboon

1570528326 Development of Behavior Monitoring System for Honeybees in Hive Using RFID sensors and Image Processing by Shinya Takahashi, Koji Hashimoto, Sakashi Maeda, Yujie Li, Naoyuki Tsuruta and Hiroyuki Ai

1570536960 Analysis and Prediction of Temporal Twitter Popularity Using Dynamic Time Warping

by Rattasit Sermsai and Sirisup Laohakiat

1570537018 Text Generation for Imbalanced Text Classification

by Suphamongkol Akkaradamrongrat, Pornpimon Kachamas and Sukree Sinthupinyo

1570537322 Information Extraction based on Named Entity for Tourism

Corpus

by Chantana Chantrapornchai and Apisit Tunsakul

1570537371 Cross-Category Product Recommender System based on Multi-Criteria Rating using Diversity and Novelty Evaluation by Saranya Maneeroj, Pongsakorn Jirachanchaisiri, Chanisara Suksomjit and Apirom Zatloukal 


\section{$\begin{array}{lll}\text { Paper ID } & \text { Title Page }\end{array}$}

1570538865 Physically-Based Modelling and Simulation of Track-based

Main Battle Tank System for a realistic 3D Game

by Yodthong Rodkaew

1570539829 Vehicle Logo Detection Using Sliding Windows with Sobel Edge Features and Recognition Using SIFT Features

by Jatupon Benjaprakairat and Pakorn Watanachaturaporn

1570541149 Fake News Detection System using Article Abstraction

by Kyeong-hwan Kim and Chang-Seong Jeong

1570542302 An Individual Local Mean-based 2DPCA for Face Recognition under Illumination Effects

by Kangsadan Hancherngchai, Taravichet Titijaroonroj and Jaratsri Rungrattanaubol

1570542304 Modified Scale-Space Analysis in Frequency Domain Based on Adaptive Multiscale Gaussian Filter for Saliency Detection by Jenjira Jaemsiri, Taravichet Titijaroonroj and Jaratsri Rungrattanaubol

1570542305 Seven Segment Display Detection and Recognition usin

Pre-defined HSV Color Slicing Technique by Sorawee Popayorm, Taravichet Titijaroonroj, Thanathorn Phoka and Wansuree Massagram

1570542312 Quantitative Trading Machine Learning Using Differential by Napas Vinitnantharat, Narit Incha, Thatthai Sakkumjor, Kitsada Doungjitjaroen and Chukiat Worasucheep

1570542529 Ensemble CNN and MLP with Nurse Notes for Intensive Care Unit Mortality by Aye Hninn Khine, Wiphada Wettayaprasit and Jarunee Duangsuwan

1570542534 Convolutional Neural Networks Using MobileNet for Skin Lesion Classification

by Wannipa Sae-Lim, Wiphada Wettayaprasit and

Pattara Aiyarak 


\section{$\begin{array}{lll}\text { Paper ID } & \text { Title Page }\end{array}$}

1570542693 A Hotel Hybrid Recommendation Method based on Context- 242 Driven using Latent Dirichlet Allocation

by Weraphat Nimchaiyanan and Saranya Maneeroj

1570542719 Spatio-Temporal Deep Learning for Ocean Current Predic- 248 tion Based on HF Radar Data

by Nathachai Thongniran, Peerapon Vateekul, Kulsawasd Jitkajornwanich, Siam Lawawirojwong and Panu Srestasathiern

1570542737 Utilizing Google Translated Reviews from Google Maps in Sentiment Analysis for Phuket Tourist Attractions

by Boonyanit Mathayomchan and Kunwadee Sripanidkulchai

1570542740 Thai Sign Language Recognition Using 3D Convolutional Neural Networks

by Nutisa Sripairojthikoon and Jaturon Hansomboon

1570542979 Identifying an Original Copy of The Source Codes in Programming Assignments

by Chawalit Saoban and Sunisa Rimcharoen

1570543012 Classification of Nutrient Deficiency in Black Gram Using

Deep Convolutional Neural Networks

by Kadipa Aung Myo Han and Ukrit Watchareeruetai

1570543029 An Open-source Based Automatic Car Detection System using

IoT

by Assadarat Khurat, Nappaphol Siriphun,

Jiratchaya Saingthong and Jirapat Sriwiphasathit

1570543035 Natural Language Contents Evaluation System for Detecting

Fake News using Deep Learning

by Ye-chan Ahn and Chang-Sung Jeong

1570543077 A Hybrid Engine for Clinical Information Extraction from Radiology Reports

by Er. Khushbu Gupta, Ratchainant Thammasudjarit and

Ammarin Thakkinstian 


\begin{tabular}{|c|c|c|}
\hline Paper ID & Title & Page \\
\hline 1570543082 & $\begin{array}{c}\text { Classification of Anger Voice in Call Center Dialog } \\
\text { by Widakorn Saewong and Janjao Mongkolnavin }\end{array}$ & 292 \\
\hline 1570547230 & $\begin{array}{l}\text { Classification of Fruit In a Box (FIB) Using Hybridization of } \\
\text { Color and Texture Features } \\
\text { by Jirapat Watcharasing, Thanaporn Thiralertphanich, Sasipa } \\
\text { Panthuwadeethorn and Suphakant Phimoltares }\end{array}$ & 297 \\
\hline 1570547250 & $\begin{array}{l}\text { Graph Clustering with K-Nearest Neighbor Constraints } \\
\text { by Wararat Jakawat and Raywat Makkhongkaew }\end{array}$ & 303 \\
\hline 1570547316 & $\begin{array}{l}\text { Optimizing a Number of Overlapping Items for Eqauting Es- } \\
\text { timated Item Parameters } \\
\text { by Sarunya Deachnatee }\end{array}$ & 308 \\
\hline 1570547403 & $\begin{array}{l}\text { Region-Focus Training: Boosting Accuracy for Deep- } \\
\text { Learning Image Segmentation } \\
\text { by Chanok Pathompatai, Ratchadaporn Kanawong and } \\
\text { Pinyo Taeprasartsit }\end{array}$ & 313 \\
\hline 1570542506 & $\begin{array}{l}\text { An Image-Based Vocabulary Learning System Based on } \\
\text { Multi-Agent System } \\
\text { by Preecha Tangworakitthaworn, Preeyapol Owatsuwan, } \\
\text { Nutsima Nongyai and Nongnapas Arayapong }\end{array}$ & 318 \\
\hline 1570542924 & $\begin{array}{l}\text { Software Defect Detection Based On Selected Complexity Met- } \\
\text { rics Using Fuzzy Association Rule Mining and Defective Mod- } \\
\text { ule Oversampling } \\
\text { by Mohammad Naufal and Selvia Kusuma }\end{array}$ & 324 \\
\hline 1570546325 & $\begin{array}{l}\text { Automatic Question Generation With Classification Based On } \\
\text { Mind Map } \\
\text { by Selvia Ferdiana Kusuma, Daniel Oranova Siahaan, } \\
\text { Chastine Fatichah and Mohammad Farid Naufal }\end{array}$ & 330 \\
\hline 1570547008 & $\begin{array}{l}\text { User Story Extraction from Online News for Software Re- } \\
\text { quirements Elicitation: A Conceptual Model } \\
\text { by Indra Kharisma Raharjana, Daniel Siahaan and } \\
\text { Chastine Fatichah }\end{array}$ & 336 \\
\hline
\end{tabular}




\section{Paper ID Title Page}

1570547291 Sequence Diagram Similarity Measurement: A Different Ap- 342 proach

by Evi Triandini, Reza Fauzan, Daniel O Siahaan and Siti Rochimah

1570537935 A Classification for Patients with Heart Disease Based on Ho- 346 effding Tree

by Sattarpoom Thaiparnit, Sorratha Kritsanasung and

Narumol Chumuang

1570541402 The Method of Integrating Virtual Reality with Brainwave

by Erdhi Widyarto Nugroho and Bernardinus Harnadi

1570543027 Impacts of Camera Frame Pacing for Video Recording on Time-Related Applications

by Nattapong Tangjui and Pinyo Taeprasartsit

1570543053 IoT-based Seven Segment Display Reader with Chessboard

Calibration and Template Determination

by Wansuree Massagram and Thanathorn Phoka

1570543059 Feature Reduction from Correlation Matrix for Classification of Two Basil Species in Common Genus

by Varin Chouvatut and Supawit Wattanapairotrat

1570547314 Eye-Tracking Based Visualizations and Metrics Analysis for Individual Eye Movement Patterns

by Rasa Bhattarai and Montri Phothisonothai

1570547322 Implementation the SoC of PCB Reflow Soldering

by Thanat Sooknuan 